\title{
Factores críticos para la adopción de las TIC en micro y pequeñas empresas industriales
}

Edgar Fernando Vasquez Reyes ${ }^{1}$

\begin{abstract}
RESUMEN
Las micro y pequeñas empresas emplean aproximadamente al $60 \%$ de la población económicamente activa (PEA) ocupada en nuestro país, pero solo el $15 \%$ de ellas tiene acceso a las tecnologías de la información y la comunicación (TIC). Por ese motivo se percibe que no comprenden estas herramientas tecnológicas, no les dan el valor correspondiente ni la importancia debida y, como consecuencia de ello, son lentas en la adopción y uso de las TIC. La presente investigación de tipo no experimental busca evidencia de la relación entre factores críticos y la adopción de las tecnologías de la información en este tipo de empresas. Para ello se realiza una encuesta a 179 empresarios en Lima Metropolitana. Luego de la evaluación y análisis de los datos obtenidos, se demuestra que 11 factores tienen una correlación positiva para la adopción de tecnologías de la información y 3 se rechazan porque el nivel de significancia obtenido es mayor a 0.05 . Así, se genera un nuevo modelo de adopción de tecnologías de la información para este tipo de empresas basado en los 11 factores aceptados.
\end{abstract}

Palabras clave: mype; TIC; TOE; adopción de tecnologías; industria manufacturera.

\section{INTRODUCCIÓN}

En la actualidad, todas las organizaciones (grandes y pequeñas) se enfrentan a situaciones relevantes: nuevas tendencias de gestión, uso de herramientas tecnológicas, software más avanzado, multicanalidad y omnicanalidad, etc. Sin embargo, se observa que, en particular, las mypes no aceptan y no utilizan las herramientas TIC, lo que les impide aprovechar las oportunidades de crecimiento potencial. En su Encuesta Económica Anual 2018, El Instituto Nacional de Estadística e Informática evidencia que $97.7 \%$ de la industria manufacturera usan computadoras para su trabajo diario. El $91.8 \%$ de las MYPE usa internet. Adicionalmente el $59.2 \%$ de las empresas usan el correo electrónico y chat como principal herramienta tecnológica para comunicarse con su personal, clientes y proveedores (INEI, 2020).

Para conocer esta realidad en el Perú, es importante realizar más investigaciones que identifiquen los factores críticos o determinantes que limitan la adopción de las TIC en las mypes industriales de Lima Metropolitana. En el último quinquenio, un sin fin de investigaciones relacionadas con la adopción de tecnologías de la información han hecho referencia a los beneficios de las TIC y una variedad de factores críticos que influyen en su adopción.

En el 2016, Cuevas-Vargas, Estrada, y Larios-Gómez (2016), argumentaron que las TIC contribuyen a mejorar las economías, generar crecimiento y eficiencia, y facilitar la transformación digital a través de las mejoras de procesos y automatismo. Hassan (2017) estudió el efecto de los factores organizativos en la adopción de cloud computing (computación en la nube) entre las pymes del sector de servicios en Malasia. Los resultados obtenidos en su estudio indicaron que el factor crítico recursos de TI tuvo una influencia significativa en la adopción de cloud computing; sin embargo, los factores críticos como el apoyo de la alta dirección y el conocimiento de los empleados no fueron significativos.

1 Ingeniero empresarial y de sistemas de la Universidad San Ignacio de Loyola. Actualmente, es Jefe de Proyectos de TI en Stefanini Peru S.A. (Lima, Perú).

ORCID: https://orcid.org/0000-0002-7220-1675

E-mail: edgarfernando.vasquez@unmsm.edu.pe 
Por su parte, Singh, Luthra, Mangla, y Uniyal (2019) realizaron un estudio de las pymes del sector alimentario, en el cual propusieron 17 factores críticos para la adopción de tecnologías. En su estudio indicaron que las organizaciones en el sector de los alimentos estaban tratando de poner en uso las TIC para reestructurar las actividades de la cadena de valor con el objetivo de reducir el desperdicio, lograr una mejor utilización de los recursos y proteger el medio ambiente.

Chau y Deng (2018) desarrollaron un modelo conceptual que les permite a los investigadores probar y comprender empíricamente los factores críticos para la adopción del comercio móvil en las pymes vietnamitas. Este modelo permite evaluar los siguientes factores críticos: beneficios percibidos, compatibilidad percibida, complejidad percibida, seguridad percibida, costos percibidos, conocimiento de TI de los empleados, preparación organizacional, orientación estratégica, soporte de la alta dirección, presión competitiva, presión del cliente y apoyo del gobierno. En Vietnam, los dispositivos móviles son el medio más popular de conexión a Internet, dado que representan el $98 \%$ de los dispositivos utilizados para acceder a Internet. Sin embargo, la lenta adopción del comercio móvil en las pymes vietnamitas puede atribuirse a muchos desafíos. Para empezar, las pymes vietnamitas comparten las mismas barreras para la adopción de nuevas tecnologías en general y del comercio móvil en particular que las pymes de otros países en desarrollo.

En la actualidad, muchas de las micro y pequeñas empresas se han visto forzadas a efectuar el cierre de sus operaciones como resultado de la pandemia del COVID-19, dado que no cuentan con tecnología para operar online. Las redes sociales se han visto inundadas con ofertas de bienes y servicios con un fuerte componente de manejo digital. Las micro y pequeñas empresas se han visto obligadas a adoptar esta forma de atención de clientes que es nueva para ellas, pues antes no tenían el interés de hacerlo. Esta investigación busca identificar los factores críticos que impedían que adoptaran dicha forma de trabajo.

Para reforzar ese conocimiento, en este trabajo de investigación centrado en las micro y pequeñas empresas, se plantean dos objetivos: proponer un modelo conceptual coherente que incluya los factores críticos más relevantes para el éxito de la adopción de las TIC y probar el modelo propuesto recopilando datos cuantitativos con base en sus atributos críticos.
Además, se plantea también la siguiente hipótesis general: Existe relación entre el modelo propuesto y la adopción de las TIC en las mypes industriales en Lima Metropolitana.

Este estudio presenta algunas limitaciones, dado que se centra en la etapa de desarrollo y revisión del modelo conceptual, por lo tanto, será necesario realizar un estudio experimental más adelante para probar la propuesta y confirmar los resultados.

Debido a las limitaciones de la investigación sobre la adopción de tecnologías de información en las mypes, los factores críticos específicos en el modelo propuesto para la adopción de las TIC se identificaron a través de una investigación cualitativa. La población del estudio estuvo conformada por las mypes que no habían adoptado las TIC localizadas en la Zona 4, distrito de La Victoria de Lima-Metropolitana, de acuerdo con la clasificación APEIM NSE 2020. Las diferencias en su percepción sobre los factores críticos pueden crear el sesgo para los resultados empíricos. Finalmente, el marco propuesto se desarrolló para el caso de las mypes, localizadas en Lima Metropolitana, que aún no adoptan un sistema de información.

Se propone un modelo integrador que permite resumir las principales contribuciones de los estudios previos, en donde se han identificado los factores críticos para la adopción de las TIC en las mypes industriales de Lima Metropolitana. La base teórica de la adopción de tecnología a nivel de organización describe cómo el contexto de la empresa influye en la adopción e implementación de innovaciones tecnológicas.

Numerosos estudios empíricos han analizado los atributos críticos de éxito para la adopción de las TIC. En este estudio, se realizó una exhaustiva revisión de la literatura relevante en un esfuerzo por obtener una visión completa del tema y, por consiguiente, definir mejor su alcance. De la literatura revisada se desglosan los atributos presentados en la Tabla 1.

El modelo propuesto se muestra en la Figura 1.

El estudio contribuye a fortalecer el conocimiento de los factores críticos para la adopción de las TIC en las mypes y ayuda a los gerentes a mejorar la evaluación de la condición de la empresa y los posibles factores críticos que conducirían a la adopción exitosa de las TIC en sus empresas.

\section{METODOLOGÍA}

De acuerdo a la tipología de Hernández (2014), este estudio es de tipo descriptivo - correlacional. 
Tabla 1. Atributos críticos identificados.

\begin{tabular}{lll}
\hline \multicolumn{1}{c}{ Factor } & \multicolumn{1}{c}{ Atributo CE } & \multicolumn{1}{c}{ Referencia literaria } \\
\hline Tecnológico & Beneficios percibidos & (Oliveira y Martins, 2010), (Chau y Deng, 2018) \\
& $\begin{array}{l}\text { Complejidad percibida } \\
\text { Seguridad percibida }\end{array}$ & $\begin{array}{l}\text { (Rogers y Shoemaker, 1971), (Thong, 1999), (Chau y Deng, 2018) } \\
\text { (Sulaiman, 2000), }\end{array}$ \\
& Costo percibido & $\begin{array}{l}\text { (Thong, 1999), ( Chang, Hung, Yen, y Lee, 2010), (Tornatzky y } \\
\text { Fleischer, 1990) }\end{array}$ \\
\hline Organizacional & Preparación tecnológica & (Rahayu y Day, 2015), (Zhu, Kraemer, y Xu, 2006), \\
& Experiencia tecnológica previa & (Raut, Gardas, Jha, y Priyadarshinee, 2017) \\
& La resistencia al cambio & (Luiz, Costa, Dos Santos, y Tsutsumi, 2020) \\
& La cultura organizacional & (Chiavenato, 2002), (Gibson, Ivancevich, Donelly, y Konospake, 2006) \\
\hline Entorno o & Presión de los proveedores & (Rahayu y Day, 2015), (Duan, Deng, y Corbitt, 2012) \\
Ambiental & Presión de los competidores & (Zhu y Kenneth, 2005) \\
& Apoyo gubernamental & (Rauta, Gardas, Jha, y Priyadarshinee, 2017), (Alrawabdeh, 2014) \\
\hline Individual & Compromiso de la alta gerencia & (Thong, 1999), (Rauta, Gardas, Jha, y Priyadarshinee, 2017), \\
& Actitudes de los gerentes hacia la & (Gangwar, Date, Ramaswamy, 2015) \\
& adopción de TI & (Chang, Hung, Yen, y Lee, 2010), (Chen, Windasari, y Pai, 2013) \\
& Experiencia TIC de la gerencia & (Rahayu y Day, 2015), (Morteza, Arias-Aranda, y Benitez-Amado, 2011) \\
\hline
\end{tabular}

Fuente: Elaboración propia.

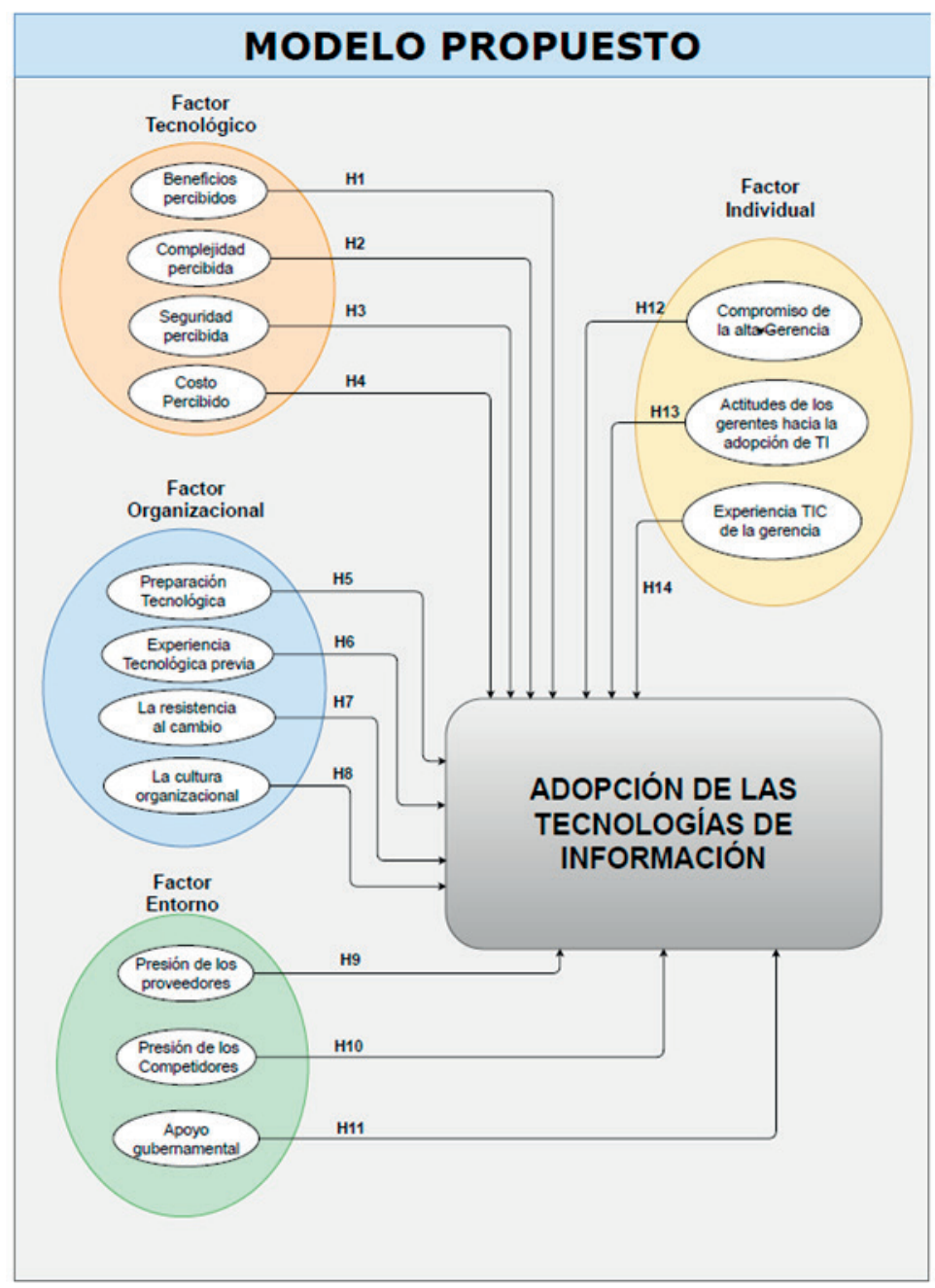

Figura 1. Modelo propuesto.

Fuente: Adaptado de Tornatzky y Fleischer (1990). 
Es descriptivo porque se especifican las propiedades y características de personas y se las somete a análisis. Es correlacional porque se calcula el grado de relación que existe entre dos o más variables. Se aplica el diseño no experimental, transeccional, correlacionales-causales, dado que no se modifican deliberadamente las variables, solo se visualizan y analizan los sucesos en su ambiente natural para describir las variables evaluadas en términos correlacionales.

La unidad de análisis de la investigación está constituida por los propietarios, gerentes, jefes o empleados con experiencia laboral en una micro, pequeña o mediana empresa industrial en Lima Metropolitana.

El instrumento de recolección de datos es la encuesta y los resultados serán procesados con el programa estadístico SPSS versión 25.0 para Windows y Excel 2019 para procesar las tablas.

En la Figura 2, se observa la población polinomial. Se muestra la cantidad de empresas industriales en Lima Metropolitana que asciende a un total de 99 477, de las cuales 92369 son micro empresas y 7 108 son medianas empresas.

Sobre la base de las 99477 empresas identificadas, se aplicó la siguiente segmentación tomando como referencia a las empresas industriales que pertenecen a la Zona 4, distrito de La Victoria, de acuerdo a la clasificación APEIM NSE 2020 y que no habían adoptado las TIC. La población estuvo conformada por 186 mypes. La desviación estándar $(\sigma)$ se calculó con una encuesta piloto a un grupo de 67 encuestados que arrojó como resultado más pesimista 1.71 , un margen de error de $5 \%$ y un nivel de confianza de $95 \%$.

En la Tabla 2, se presenta el cuadro para el cálculo de la muestra:

Tabla 2. Parámetros para el cálculo de muestra.

\begin{tabular}{lcc}
\hline Descripción & Variable & Valor \\
\hline Tamaño de la población & $\mathrm{N}$ & 186 \\
Nivel de confianza & $\mathrm{Z}$ & 1.96 \\
Desviación estándar & $\sigma$ & 1.71 \\
Error de estimación & $\mathrm{e}$ & 0.05 \\
\hline
\end{tabular}

Fuente: Elaboración propia.

Fórmula para calcular el tamaño de la muestra:

$$
\frac{N Z^{2} \sigma^{2}}{e^{2}(N-1)+Z^{2} \sigma^{2}}
$$

La muestra estuvo compuesta por 179 individuos.

La fiabilidad del instrumento se comprobó mediante la aplicación de una encuesta piloto. Inicialmente, se identificaron 67 preguntas para el instrumento a utilizar y se evaluaron indicadores estadísticos que permitieran validar y ajustar el instrumento final. Con estas preguntas se realizó la encuesta inicial en marzo de 2020 de forma virtual. En un principio, se pensó realizar la encuesta de forma mixta, es decir, física y virtual, en los principales centros de producción industrial de Lima Metropolitana que no habían adoptado las TIC y pertenecen a la Zona

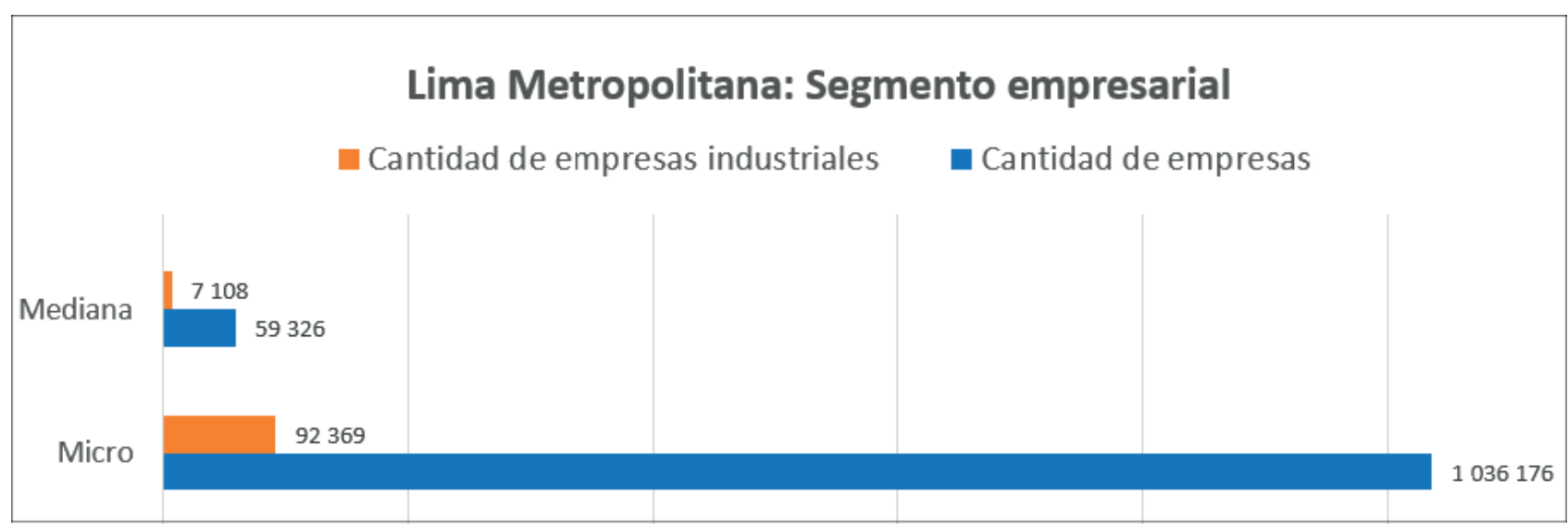

Figura 2. Población del estudio. Fuente: Elaboración propia. 
4 - distrito de la Victoria. Para ello, se conformó un equipo de 10 personas, incluido el investigador; sin embargo, la encuesta de forma física tuvo que ser descartada, debido a la pandemia que azota el planeta en el año 2020.

En la encuesta piloto, de los 36 cuestionarios completados, solo 28 fueron válidos. Luego se calculó el Alfa de Cronbach y se eliminaron 13 preguntas porque no alcanzaron el valor esperado. Finalmente, se identificaron 54 preguntas para evaluar la adopción de tecnologías de la información en las mypes industriales de Lima Metropolitana y, adicionalmente, 8 preguntas generales.

En la Tabla 3, se muestran los resultados de confiabilidad para el instrumento obtenidos mediante el coeficiente alfa de Cronbach.

Se consideraron 54 preguntas para el instrumento de encuesta final tras depurar 13 preguntas del cuestionario inicial; los ítems finales obtuvieron el indicador de Alpha de Cronbach esperado.

\section{Variables identificadas}

Independiente: Factores críticos de éxito

Dependiente: Adopción de las Tecnologías de la Información en las mypes industriales

\section{RESULTADOS}

\section{Encuesta final}

La encuesta final se realizó con 62 ítems, 8 ítems de control y 54 ítems para la investigación, estas últimas divididas en 14 atributos críticos o constructos según el modelo propuesto para la investigación, se obtuvieron 190 encuestas validas de 210 realizadas. A las personas responsables dentro de la empresa se les envió vía comunicación electrónica (email, WhatsApp, otro) el link de la encuesta con una guía de llenado. Los resultados serán procesados con el programa estadístico SPSS versión 25.0 para Windows y Excel 2019 para generar las tablas (Tabla 4).

Tomando en cuenta que el cuestionario cuenta con 54 ítems y 14 constructos (atributos críticos), fue necesario someter cada constructo a un análisis factorial exploratorio para determinar su adecuada formación. Esto se observa en la Tabla 5.

Como se observa en la Tabla 5, tras realizar el análisis factorial de los constructos se determina que son pertinentes; además, con base en el modelo original, es posible crear grupos de constructos más homogéneos según su correlación e interdependencia.

Tabla 3. Fiabilidad del documento.

\begin{tabular}{|c|c|c|c|c|c|}
\hline Atributo CE & $\begin{array}{c}\text { Alfa de Cronbach } \\
\text { inicial }\end{array}$ & $\begin{array}{c}\text { Alfa de Cronbach } \\
\text { final }\end{array}$ & $\begin{array}{c}N .{ }^{\circ} \text { de ítems } \\
\text { iniciales }\end{array}$ & $\begin{array}{c}N .^{\circ} \text { de ítems de- } \\
\text { purados }\end{array}$ & $\begin{array}{c}N .{ }^{\circ} \text { de ítems } \\
\text { finales }\end{array}$ \\
\hline VO & 0.726 & 0.914 & 8 & 2 & 6 \\
\hline V1 & 0.900 & 0.900 & 8 & & 8 \\
\hline V2 & 0.592 & 0.899 & 7 & 4 & 3 \\
\hline V3 & 0.906 & 0.906 & 3 & & 3 \\
\hline V4 & 0.716 & 0.756 & 4 & 1 & 3 \\
\hline V5 & 0.919 & 0.919 & 3 & & 3 \\
\hline V6 & 0.504 & 0.880 & 6 & 3 & 3 \\
\hline V7 & 0.796 & 0.796 & 3 & & 3 \\
\hline V8 & 0.824 & 0.824 & 3 & & 3 \\
\hline V9 & 0.660 & 0.660 & 3 & & 3 \\
\hline V10 & 0.681 & 0.737 & 5 & 2 & 3 \\
\hline V11 & 0.825 & 0.825 & 3 & & 3 \\
\hline V12 & 0.853 & 0.853 & 3 & & 3 \\
\hline V13 & 0.889 & 0.889 & 4 & & 4 \\
\hline V14 & 0.751 & 0.801 & 4 & 1 & 3 \\
\hline TOTAL & & & 67 & 13 & 54 \\
\hline
\end{tabular}

Fuente: Elaboración propia. 
Tabla 4. Fiabilidad de la herramienta final.

\begin{tabular}{llcc}
\hline Atributo CE & Preguntas de control & Alfa de Cronbach & Ítems \\
\hline V0 & Sobre la adopción de tecnologías de la información & 0.891 & 6 \\
V1 & Beneficios percibidos & 0.943 & 8 \\
V2 & Complejidad percibida & 0.882 & 3 \\
V3 & Seguridad percibida & 0.908 & 3 \\
V4 & Costo percibido & 0.914 & 3 \\
V5 & Preparación tecnológica & 0.839 & 3 \\
V6 & Experiencia tecnológica previa & 0.860 & 3 \\
V7 & Resistencia al cambio & 0.995 & 3 \\
V8 & Cultura organizacional & 0.866 & 3 \\
V9 & Presión de los proveedores & 0.841 & 3 \\
V10 & Presión de los competidores & 0.814 & 3 \\
V11 & Apoyo gubernamental & 0.857 & 3 \\
V12 & Compromiso de la alta gerencia & 0.988 & 3 \\
V13 & Actitudes de los gerentes hacia la adopción de TIC & 0.854 & 4 \\
V14 & Experiencia TIC de la gerencia & 0.832 & 3 \\
\hline V1-14 & & $\mathbf{0 . 9 6 1}$ & $\mathbf{5 4}$ \\
\hline
\end{tabular}

Fuente: Elaboración propia.

Tabla 5. Matriz de componente rotado.

\begin{tabular}{|c|c|c|c|c|c|c|}
\hline \multirow{2}{*}{ Atributo CE } & \multirow{2}{*}{ Descripción de la variable } & \multirow{2}{*}{ Ítems } & \multicolumn{4}{|c|}{ Correlación de ítems } \\
\hline & & & $1(\mathrm{~T})$ & 2(I) & $3(E)$ & $4(0)$ \\
\hline V9 & Presión de los proveedores & 3 & 0.973 & & & \\
\hline V8 & La cultura organizacional & 3 & 0.972 & & & \\
\hline V3 & Seguridad percibida & 3 & 0.967 & & & \\
\hline V4 & Costo percibido & 3 & 0.965 & & & \\
\hline V2 & Complejidad percibida & 3 & 0.956 & & & \\
\hline V1 & Beneficios percibidos & 8 & 0.667 & & & \\
\hline V13 & Actitudes de los gerentes hacia la adopción de TIC & 4 & & 0.845 & & \\
\hline V12 & Compromiso de la alta gerencia & 3 & & 0.752 & & \\
\hline V11 & Apoyo gubernamental & 3 & & 0.694 & & \\
\hline V5 & Preparación tecnológica del personal & 3 & & 0.587 & & \\
\hline V10 & Presión de los competidores & 3 & & & 0.860 & \\
\hline V6 & Experiencia tecnológica previa & 3 & & & 0.798 & \\
\hline V7 & La resistencia al cambio & 3 & & & & 0.853 \\
\hline V14 & Experiencia TIC de la gerencia & 3 & & & & 0.740 \\
\hline
\end{tabular}

Método de extracción: análisis de componentes principales.

Método de rotación: Varimax con normalización Kaiser.

Fuente: Elaboración propia.

El modelo de la nueva distribución de atributos se muestra en la Figura 3.

Por el tipo de modelo propuesto en la investigación es necesario trabajar con base en los 14 atributos. En la Tabla 4, se aprecia la consistencia interna de cada uno de ellos, todos los atributos tienen un coeficiente alfa de Cronbach superior a 0.81 ; es decir, las consistencias internas son las adecuadas.

En total, el coeficiente alfa de Cronbach de los 54 ítems es de 0.961, lo que demuestra que tienen una consistencia muy buena en conjunto (Tabla 6). 


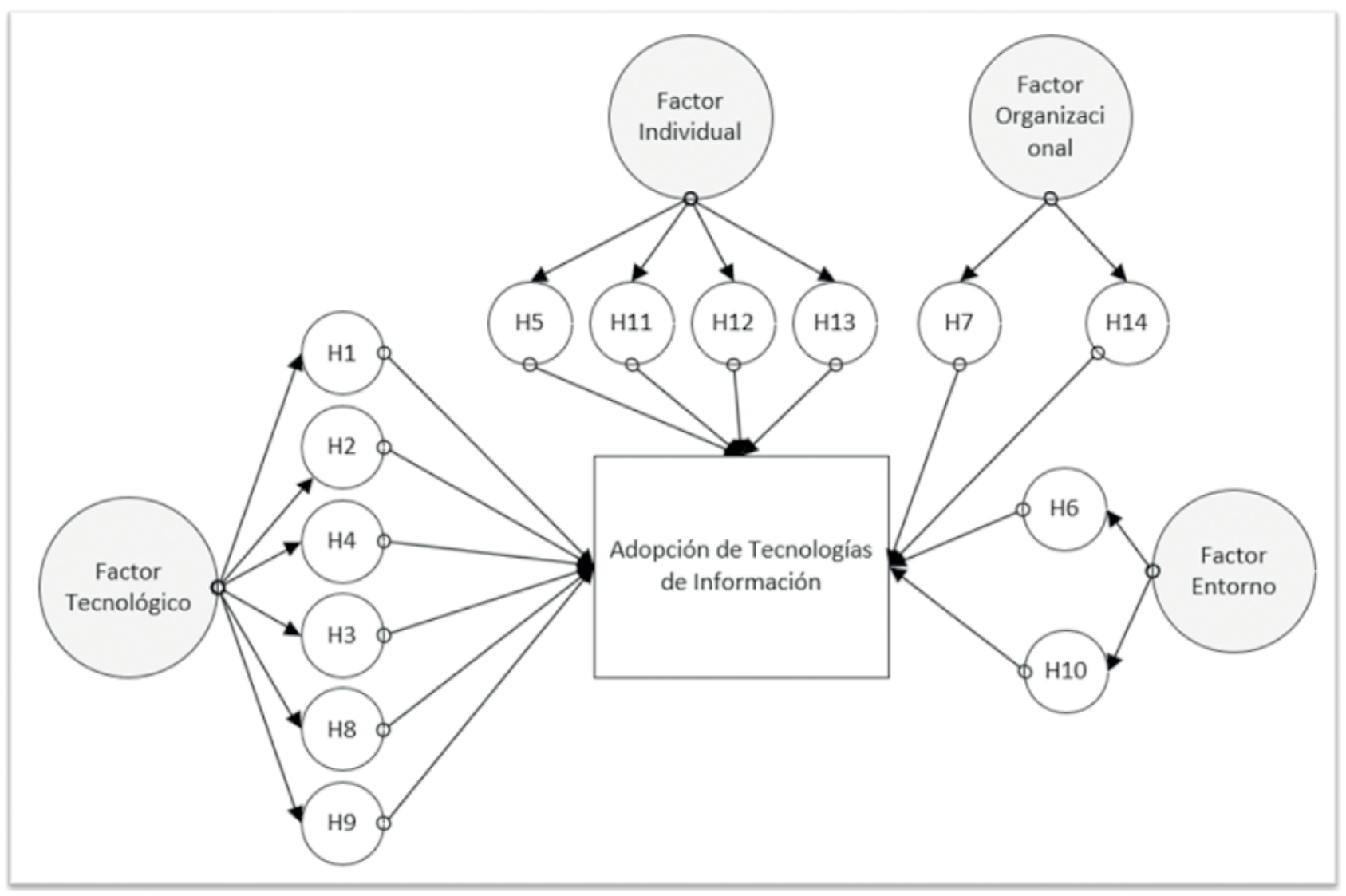

Figura 3. Modelo - Análisis factorial.

Fuente: Elaboración propia.

Tabla 6. Resumen resultado prueba de hipótesis.

\begin{tabular}{|c|c|c|c|c|}
\hline & Atributo crítico de éxito & Sig. & & Correlación \\
\hline & Hipótesis general & 0.000 & 0.770 & Correlación positiva considerable \\
\hline V1 & Beneficios percibidos & 0.000 & 0.780 & Correlación positiva considerable \\
\hline V2 & Complejidad percibida & 0.000 & 0.670 & Correlación positiva media \\
\hline V3 & Seguridad percibida & 0.000 & 0.611 & Correlación positiva media \\
\hline V4 & Costo percibido & 0.000 & 0.606 & Correlación positiva media \\
\hline V5 & Preparación tecnológica de los usuarios & 0.000 & 0.758 & Correlación positiva considerable \\
\hline V6 & Experiencia tecnológica previa & 0.622 & 0.036 & No existe correlación alguna entre las variables \\
\hline V7 & La resistencia al cambio & 0.544 & -0.044 & No existe correlación alguna entre las variables \\
\hline V8 & La cultura organizacional & 0.000 & 0.586 & Correlación positiva media \\
\hline V9 & Presión de los proveedores & 0.000 & 0.597 & Correlación positiva media \\
\hline V10 & Presión de los competidores & 0.561 & 0.042 & No existe correlación alguna entre las variables \\
\hline V11 & Apoyo gubernamental & 0.000 & 0.564 & Correlación positiva media \\
\hline V12 & Compromiso de la alta gerencia & 0.000 & 0.553 & Correlación positiva media \\
\hline V13 & Actitudes de los gerentes hacia la adopción de TI & 0.000 & 0.772 & Correlación positiva considerable \\
\hline V14 & Experiencia TIC de la gerencia & 0.000 & 0.271 & Correlación negativa media \\
\hline
\end{tabular}

Fuente: Elaboración propia. 
Como se observa en la tabla anterior, se acepta la hipótesis general, debido a que el nivel de significancia resultante de la prueba es menor a 0.05 . Además, la hipótesis general tiene correlación positiva considerable, dado que la correlación resultante es 0.770 .

\section{Modelo final}

El modelo final se muestra en la Figura 4. Este no incluye el factor "entorno", puesto que sus atributos críticos fueron rechazados porque obtuvieron niveles de significancia mayores a 0.05 en el resultado de las hipótesis.

\section{DISCUSIÓN}

El atributo crítico experiencia TIC de la gerencia (14) se acepta porque el nivel de significancia es menor a 0.05 , con 0.271 de correlación negativa débil.

En el caso de los atributos críticos beneficios percibidos (V1), preparación tecnológica de los usuarios (V5) y actitudes de los gerentes hacia la adopción de las TIC (V13), se observa una correlación positiva considerable, dado que la correlación resultante oscila entre 0.758 y 0.780 . Por tanto, se puede decir que existe una correlación positiva considerable entre estos atributos planteados y la adopción de tecnologías de la información y comunicación en las mypes industriales de Lima Metropolitana.

En el caso de los atributos críticos seguridad percibida (V3), costo percibido (V4), cultura organizacional (V8), presión de los proveedores (V9), apoyo gubernamental (V11) y compromiso de la alta gerencia (V12), se observa una correlación positiva media, dado que la correlación resultante oscila entre 0.553 y 0.611 . Por tanto, se puede decir que existe una correlación positiva media entre estas hipótesis planteadas y la adopción de tecnologías de la información y comunicación en las mypes industriales de Lima Metropolitana.

Por otra parte, los atributos críticos experiencia tecnológica previa (V6), resistencia al cambio (V7) y presión de los competidores (V10), se rechazan porque el nivel de significancia es mayor a 0.05 .

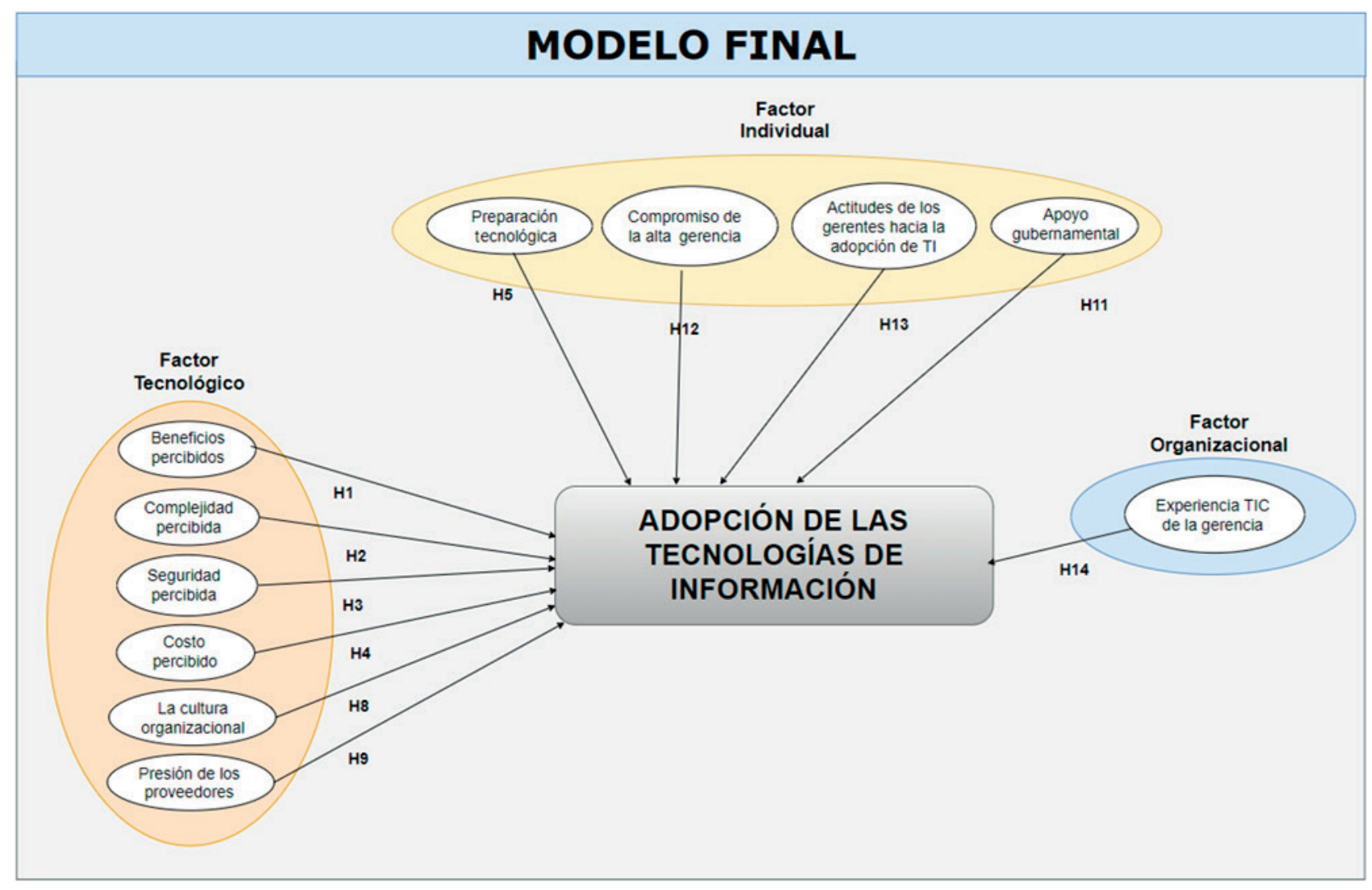

Figura 4. Modelo resultante de las hipótesis aceptadas.

Fuente: Elaboración propia. 
El marco conceptual propuesto permite a los investigadores probar y comprender empíricamente los factores críticos para la adopción de las TIC en las mypes de Lima Metropolitana. Esto es importante para la adopción exitosa de las TIC en las mypes.

Dentro de este marco propuesto, se pueden analizar los patrones actuales de adopción de las TIC y se pueden proporcionar sugerencias específicas para mejorar el modelo.

El estudio también contribuye a mejorar el conocimiento de los factores críticos para la adopción de las TIC en las mypes y ayuda a los gerentes a mejorar en la evaluación de la condición de la empresa y los posibles factores que conducirían a la adopción exitosa de las TIC en sus empresas.

Asimismo, se espera que los resultados de esta investigación contribuyan al campo académico, la pequeña y mediana empresa, las firmas consultoras y los proveedores de software y las agencias gubernamentales.

\section{CONCLUSIONES}

En esta investigación se demuestra que existe correlación entre los atributos propuestos en el modelo y la adopción de las TIC en las mypes industriales de Lima Metropolitana, lo que queda evidenciado en la Tabla 6.

Se evidencia que los atributos críticos "experiencia tecnológica previa", "resistencia al cambio" y "presión de los competidores" no tienen correlación con la adopción de tecnologías de la información y comunicación en las empresas industriales de Lima Metropolitana.

En la investigación se evidencia que el factor "entorno" desaparece en el modelo final para la adopción de tecnologías de la información y comunicación en las empresas industriales de Lima Metropolitana, dado que los atributos propuestos no son influyentes.

En la presente investigación, se analizaron catorce atributos críticos; sin embargo, puede haber otros que también son importantes y no fueron considerados en el desarrollo de este modelo.

\section{RECOMENDACIÓN}

Se recomienda aplicar el modelo final a las Zonas restantes del APEIM 2020, según industria y naturaleza de los productos que se desarrollan con la finalidad de evaluar el modelo y analizar los resultados.

\section{REFERENCIAS BIBLIOGRÁFICAS}

[1] Alrawabdeh, W. (2014). Environmental Factors Affecting Mobile Commerce Adoption-An Exploratory Study on the Telecommunication Firms in Jordan. International Journal of Business and Social Science. 5 (8)., 151-164.

[2] Chang, S., Hung, S., Yen, D., y Lee, P. (2010). Critical Factors of ERP Adoption for Small- and Medium- Sized Enterprises: An Empirical Study. Journal of Global Information Management (JGIM), 18(3), 82106. Recuperado de http://doi.org/10.4018/ jgim.2010070104

[3] Chau, N., y Deng, H. (2018). Critical Determinants for Mobile Commerce Adoption in Vietnamese SMEs: A Conceptual Framework. Procedia Computer Science, 138, 433-440. Recuperado de https://doi.org/10.1016/j. procs.2018.10.061

[4] Chen, J., Windasari, N., y Pai, R. (2013). Exploring E-Readiness on E-Commerce Adoption of SMEs: Case Study SouthEast Asia. IEEE International Conference on Industrial Engineering and Engineering Management. Recuperado de https://doi. org/10.1109/IEEM.2013.6962637

[5] Chiavenato, I. (2002). Introducción a la teoría general de la administración. $5^{2}{ }^{a}$ ed. . México D. F.: McGraw-Hill.

[6] Cuevas-Vargas, H., Estrada, S., y LariosGómez, E. (2016). The effects of ICTs as innovation facilitators for a greater business performance. Evidence from Mexico. Procedia Computer Science, 91, 47-56. Recuperado de https://doi.org/10.1016/j.procs.2016.07.040

[7] Duan, X., Deng, H., y Corbitt, B. (2012). What drives the adoption of electronic markets in Australian small-and-medium sized enterprises? - An empirical study. ACIS 2012 Proceedings. 20. Recuperado de https://aisel. aisnet.org/acis2012/20

[8] Gangwar, H., Date, H., y Ramaswamy, R. (2015). Understanding determinants of cloud computing adoption using an integrated TAMTOE model. Journal of Enterprise Information Management, 28 (1), 107-130. Recuperado de https://doi.org/10.1108/JEIM-08-2013-0065

[9] Gibson, J., Ivancevich, J., Donelly, J., y Konospake, R. (2006). Organizaciones: Comportamiento, Estructura y Procesos. 12. ${ }^{a}$ ed. México, D. F.: Mc-Graw Hill. 
[10] Hassan, H. (2017). Organisational factors affecting cloud computing adoption in small and medium enterprises (SMEs) in service sector. Procedia Computer Science, 121, 976981. Recuperado de https://doi.org/10.1016/j. procs.2017.11.126

[11] Hernández, R., Fernández, C., y Baptista, P. (2014). Metodología de la investigación (6a. ed.). México D.F.: McGraw-Hill.

[12] INEI (2020). PERÚ: Tecnologías de Información y Comunicación en las Empresas, 2017. Encuesta Económica Anual 2018. Recuperado de https://www.inei.gob.pe/media/ MenuRecursivo/publicaciones_digitales/Est/ Lib1719/libro.pdf

[13] Luiz, A., Costa, C., Dos Santos, J., y Tsutsumi, M. (2020). Resistance to change as function of different physical-effort requirements in humans. Behavioural Processes, 176. Recuperado de https://doi.org/10.1016/j. beproc. 2020.104123

[14] Morteza, G., Arias-Aranda, D., y BenitezAmado, J. (2011). Adoption of e-commerce applications in SMEs. Industrial Management \& Data Systems, 111(8), 1238-1269. Recuperado de https://doi.org/10.1108/02635571111170785

[15] Oliveira, T., y Martins, M. (2010). Understanding e-business adoption across industries in European countries. Industrial Management \& Data Systems, 110 (9), 1337-1354. Recuperado de https://doi. org/10.1108/02635571011087428

[16] Rahayu, R., y Day, J. (2015). Determinant Factors of E-commerce Adoption by SMEs in Developing Country: Evidence from Indonesia. Procedia - Social and Behavioral Sciences, 195, 142 - 150. Recuperado de https://doi. org/10.1016/j.sbspro.2015.06.423

[17] Rauta, R., Gardas, B., Jha, M., y Priyadarshinee, P. (2017). Examining the critical success factors of cloud computing adoption in the MSMEs by using ISM model. The Journal of High Technology Management Research. 28, 125141. Recuperado de https://doi.org/10.1016/j. hitech.2017.10.004
[18] Rogers, E., y Shoemaker, F. (1971). Communication of Innovations: A Cross-cultural Approach. Free Press, New York, NY.

[19] Singh, R., Luthra, S., Mangla, S., y Uniyal, S. (2019). Applications of information and communication technology for sustainable growth of SMEs in India food industry. Resources, Conservation \& Recycling, 147, 1018. Recuperado de https://doi.org/10.1016/j. resconrec.2019.04.014

[20] Sulaiman, A. (2000). The status of e-commerce applications in Malaysia. Information Technology for Development. 9, 153-161. Recuperado de https://doi.org/10.1080/026811 02.2000 .9525329

[21] Thong, J. (1999). An Integrated Model of Information Systems Adoption in Small Business. Journal of Management Information Systems, 15 (4), 187-214. Recuperado de https://doi.org/10.1080/07421222.1999.11518 227

[22] Tornatzky, L., y Fleischer, M. (1990). The process of technology innovation. Lexington, MA: Lexington Books.

[23] Zhu, K., y Kenneth, K. (2005). Post-Adoption Variations in Usage and Value of E-Business by Organizations. Information Systems Research. 16 (1), 61-84. Recuperado de http://dx.doi. org/10.1287/isre.1050.0045

[24] Zhu, K., Kraemer, K., y Xu, S. (2006). The Process of Innovation Assimilation by Firms in Different Countries: A Technology Diffusion Perspective on E-Business. Management Science, 52(10), 1557-1576. Recuperado de https://doi.org/10.1287/mnsc.1050.0487 


\section{Critical Factors for ICT Adoption in Industrial Sector Micro and Small Enterprises (MSEs)}

Edgar Fernando Vasquez Reyes ${ }^{1}$

\begin{abstract}
Micro and small-sized enterprises (MSEs) employ approximately $60 \%$ of the economically active population (EAP) in our country, but only $15 \%$ of them have access to information and communication technologies (ICTs). Therefore, it is believed that these companies do not understand ICTs, do not value them or give them due importance and are reluctant to adopt and use ICTs. This non-experimental research aims to find evidence of the relationship between critical factors and the adoption of information technologies in this type of company. To this end, a survey was conducted among 179 businessmen in Metropolitan Lima. Following the evaluation and analysis of the data obtained, it was found that 11 factors have a positive correlation for the adoption of information technologies and 3 are rejected because the significance level obtained is greater than 0.05 . Thus, a new model of ICT adoption based on the 11 accepted factors is created for this type of company.
\end{abstract}

Keywords: MSE; ICT; TOE; technology adoption; manufacturing industry.

\section{INTRODUCTION}

At present, all organizations (large and small) are facing significant situations: new management trends, use of technological tools, more advanced software, multichannel and omnichannel marketing, etc. However, it is observed that, in particular, micro and small-sized enterprises (MSEs) do not embrace and do not use ICT tools, which prevents them from leveraging potential growth opportunities. In its publication Encuesta Económica Anual 2018, the Instituto Nacional de Estadística e Informática (INEI) reveals that $97.7 \%$ of the manufacturing industry uses computers for their daily activities and $91.8 \%$ of MSEs use the internet. Also, $59.2 \%$ of companies use e-mail and chat as the main technological tool to communicate with their staff, customers and suppliers (INEI, 2020).

Further research is needed to identify the critical factors or determinants that limit the adoption of ICTs in industrial MSEs in Metropolitan Lima. Numerous research studies related to the adoption of information technologies in the last five years have referred to the benefits of ICTS and a variety of critical factors that influence their adoption.

Cuevas-Vargas, Estrada, and Larios-Gomez (2016) argued that ICT contributes to improving economies, generating growth and efficiency, and facilitating digital transformation through process improvements and automation. Hassan (2017) studied the effect of organizational factors on cloud computing adoption among small and medium-sized enterprises (SMEs) in the service sector in Malaysia. The results obtained in her study indicated that critical factor IT resources had a significant influence on cloud computing adoption; however, critical factors such as senior management support and employee knowledge were not significant.

Singh, Luthra, Mangla, and Uniyal (2019), on the other hand, conducted a study of SMEs in the food sector, in which they pro-

1 Business and Systems Engineer from the Universidad San Ignacio de Loyola. Currently working as IT Project Management at Stefanini Peru S.A. (Lima, Peru).

ORCID: https://orcid.org/0000-0002-7220-1675

E-mail: edgarfernando.vasquez@unmsm.edu.pe 
posed 17 critical factors for technology adoption. They found that organizations in the food sector were trying to use ICT to restructure value chain activities with the aim of reducing waste, improving resource utilization, and protecting the environment.

Chau and Deng (2018) developed a conceptual framework that allows researchers to empirically test and understand the critical factors for m-commerce adoption in Vietnamese SMEs. This model allows assessing critical factors such as perceived benefits, perceived compatibility, perceived complexity, perceived security, perceived security, perceived costs, employees' IT knowledge, organizational readiness, strategic orientation, top management support, competitive pressure, customer pressure, and government support. In Vietnam, mobile devices are the most popular means of Internet connection, accounting for $98 \%$ of all devices used for accessing the Internet. However, the slow adoption of m-commerce in Vietnamese SMEs can be attributed to many challenges. First of all, Vietnamese SMEs share the same barriers to the adoption of new technologies in general and $\mathrm{m}$-commerce in particular as SMEs in other developing countries. (Chau \& Deng, 2018, p. 435)

Presently, many MSEs have been forced to shut down their operations as a result of the COVID-19 pandemic, as they lack the technology to operate online. Social networks have been flooded with offers of goods and services with a strong digital management component. Micro and small-sized enterprises have had to adopt this form of customer service that is new to them, as previously they had no interest in doing so. This research attempts to identify the critical factors that prevented them from adopting this way of working.

It has two objectives. First, to propose a coherent conceptual model that includes the most relevant critical factors for the success of ICT adoption, and second, to test the proposed model by collecting quantitative data based on its critical attributes.

The general hypothesis states that "There is a relationship between the proposed model and ICT adoption in industrial MSEs in Metropolitan Lima".

There are some limitations to this study, as it focuses on the development and review stage of the conceptual model; therefore, an experimental study should be conducted at a later stage to test the proposal and corroborate the results.

Due to the limitations of the research on the adoption of ICTs in MSEs, the specific critical factors in the proposed model for ICT adoption were identified using a qualitative approach. The study population consisted of MSEs that had not adopted ICTs in Zone 4, the La Victoria district of Metropolitan Lima, according to the APEIM NSE 2020 classification. Differences in their perception of the critical factors may create bias for the empirical results. Finally, the proposed framework was developed for the case of SMEs located in Metropolitan Lima that have not yet adopted an IT system.

A comprehensive model is proposed to summarize the main contributions of previous studies, which have identified the critical factors for the adoption of ICTs in industrial MSEs in Metropolitan Lima. The theoretical basis of technology adoption at the organizational level describes how the enterprise's specific context influences the adoption and implementation of technology.

Numerous empirical studies have analyzed the critical success attributes (CSA) for ICT adoption. An exhaustive review of the relevant literature was conducted for this study in an effort to gain a comprehensive view of the topic and, consequently, better define its scope. A breakdown of the factors from the literature reviewed is presented in Table 1.

\section{The proposed model is shown in Figure 1.}

This research contributes to strengthen the knowledge of the critical factors for the adoption of ICTs in MSEs and helps managers to better assess the condition of the enterprise and the possible critical factors that would lead to the successful adoption of ICTs in their enterprises.

\section{METHODOLOGY}

As per the classification of Hernández (2014), this is a descriptive-correlational study. It is descriptive because the properties and characteristics of people are specified and subjected to analysis, and correlational because the degree of relationship that exists between two or more variables is calculated. A non-experimental, cross-sectional, correlational-causal design is used, since the variables are not deliberately modified, events are only observed and analyzed in their natural environment to describe the variables assessed on correlational terms.

The unit of analysis of the research is comprised of owners, managers, bosses or employees with work experience in a micro, small or medium-sized industrial enterprise in Metropolitan Lima. 
Table 1. Critical Attributes Identified.

\begin{tabular}{lll}
\hline \multicolumn{1}{c}{ Factors } & \multicolumn{1}{c}{ CSA } & \multicolumn{1}{c}{ Literary Reference } \\
\hline Technological & Perceived benefits & (Oliveira \& Martins, 2010), (Chau \& Deng, 2018) \\
& Perceived complexity & (Rogers \& Shoemaker, 1971), (Thong, 1999), (Chau \& Deng, 2018) \\
& Perceived security & (Sulaiman, 2000), \\
& Perceived cost & (Thong, 1999), (Chang, Hung, Yen, \& Lee, 2010), (Tornatzky \& Fleischer, \\
\hline Organizational & Technology readiness & (Rahayua \& Daya, 2015), (Zhu, Kraemer, \& Xu, 2006), \\
& Previous technological experience & (Rauta, Gardas, Jhac, \& Priyadarshinee, 2017) \\
& Resistance to change & (Luiz, Costa, Dos Santos, \& Tutsumi, 2020) \\
& Organizational culture & (Chiavenato, 2002), (Gibson, Ivancevich, Donelly, \& Konospake, 2006) \\
\hline Contextual or & Pressure from suppliers & (Rahayu \& Day, 2015), (Duan, Deng, \& Corbitt, 2012) \\
Environmental & Pressure from competitors & (Zhu \& Kenneth, 2005) \\
& Government support & (Rauta, Gardas, Jha, \& Priyadarshinee, 2017), (Alrawabdeh, 2014) \\
\hline Individual & Senior Management commitment & (Thong, 1999), (Rauta, Gardas, Jha, \& Priyadarshinee, 2017), (Gangwar, \\
& Managers' attitude towards IT & (Chang, Hung, Yen, \& Lee, 2010), (Chen, Windasari, \& Pai, 2013) \\
& $\begin{array}{l}\text { adoption } \\
\text { Management ICT experience }\end{array}$ & (Rahayu \& Day, 2015), (Morteza, Arias-Aranda, \& Benitez-Amado, 2011) \\
\hline
\end{tabular}

Source: Prepared by the author.

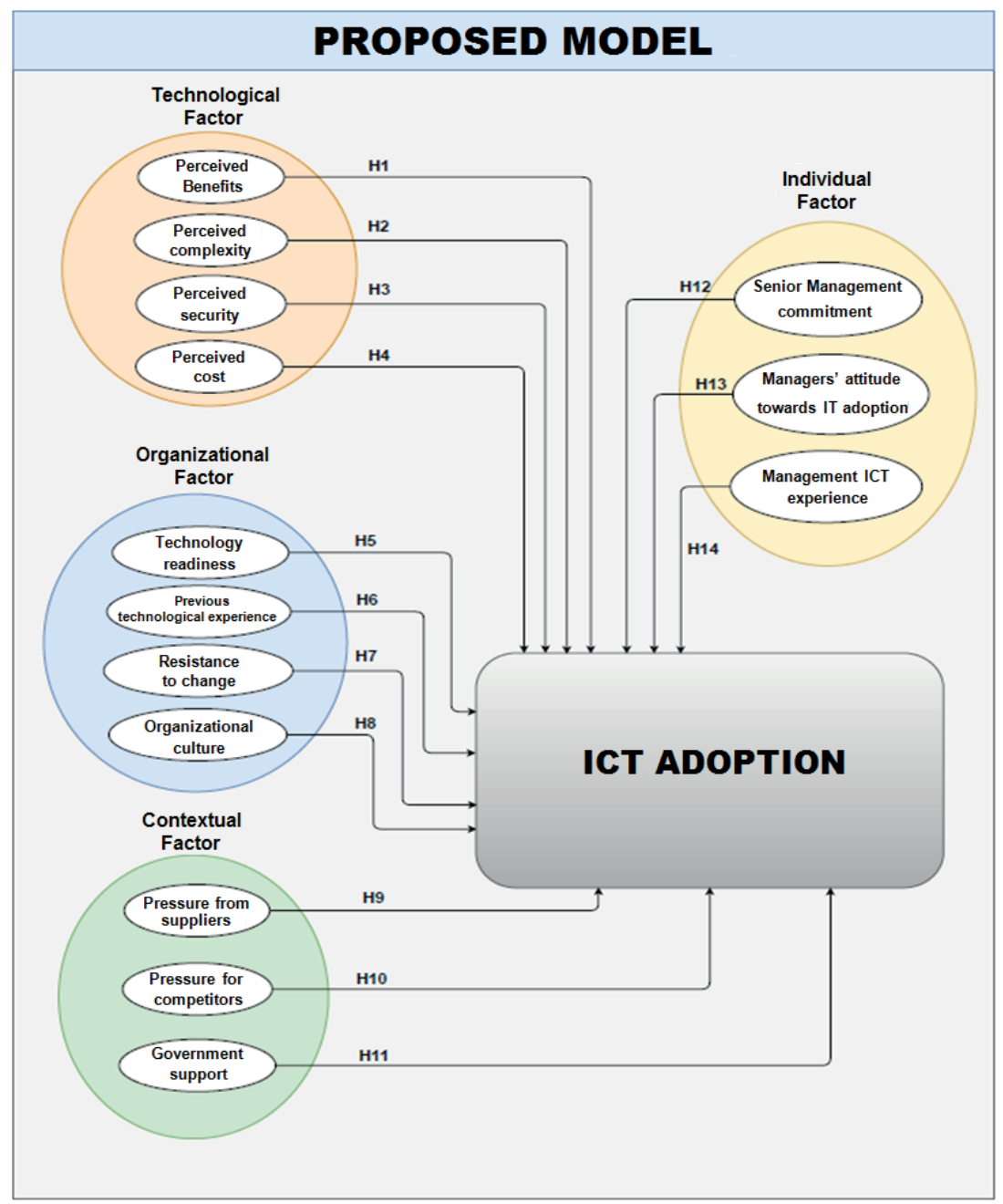

Figure 1. Proposed model.

Source: Adapted from Tornatzky and Fleischer (1990). 
The data collection instrument is the survey and the results will be processed with the statistical program SPSS version 25.0 for Windows and Excel 2019 to process the tables.

Figure 2 shows the polynomial population. It shows the number of industrial enterprises in Metropolitan Lima which totals 99 477, of which 92369 are micro enterprises and 7108 are medium-sized enterprises.

Based on the 99,477 companies identified, a segmentation was applied using as a reference the industrial enterprises in Zone 4, district of La Victoria, according to the APEIM NSE 2020 classification, that had not adopted ICTs. The population consisted of 186 MSEs. A pilot survey of a group of 67 respondents was used to calculate the standard deviation $(\sigma)$, which yielded a pessimistic result of 1.71 , a margin of error of $5 \%$ and a confidence level of $95 \%$.

The parameters used to calculate the sample are presented in Table 2:

Table 2. Parameters for Sample Calculation.

\begin{tabular}{lcc}
\hline Description & Variable & Value \\
\hline Population size & $\mathrm{N}$ & 186 \\
Confidence level & $\mathrm{Z}$ & 1.96 \\
Standard deviation & $\sigma$ & 1.71 \\
Estimation error & $\mathrm{e}$ & 0.05 \\
\hline
\end{tabular}

Source: Prepared by the author.

Sample size calculation formula:

$$
\frac{N Z^{2} \sigma^{2}}{e^{2}(N-1)+Z^{2} \sigma^{2}}
$$

There were 179 individuals in the sample.

A pilot survey was used to test the reliability of the instrument. Initially, 67 questions were selected for the instrument and statistical indicators were evaluated to validate and adjust the final instrument. The initial survey was conducted online in March 2020 using these questions. Initially, the survey was to be carried out in a mixed form, i.e. in-person and online, in the main industrial production centers of Metropolitan Lima that had not adopted ICTs and belong to Zone 4 - district of La Victoria. For this purpose, a team of 10 people was formed, including the researcher; however, the in-person survey had to be discarded, due to the 2020 pandemic.

Out of 36 questionnaires completed in the pilot survey, only 28 were valid. Cronbach's alpha was then calculated and 13 questions were eliminated because they did not reach the expected value. Finally, 54 questions were selected to evaluate the adoption of ICTs in industrial MSEs in Metropolitan Lima and, additionally, 8 general questions.

Table 3 shows the reliability results for the instrument obtained using Cronbach's alpha coefficient.

Fifty-four questions were considered for the final survey instrument after eliminating 13 questions from the initial questionnaire; the final items obtained the expected Cronbach's alpha coefficient.

\section{Variables}

Independent: Critical success factors

Dependent: Adoption of information technologies in industrial micro and small-sized enterprises

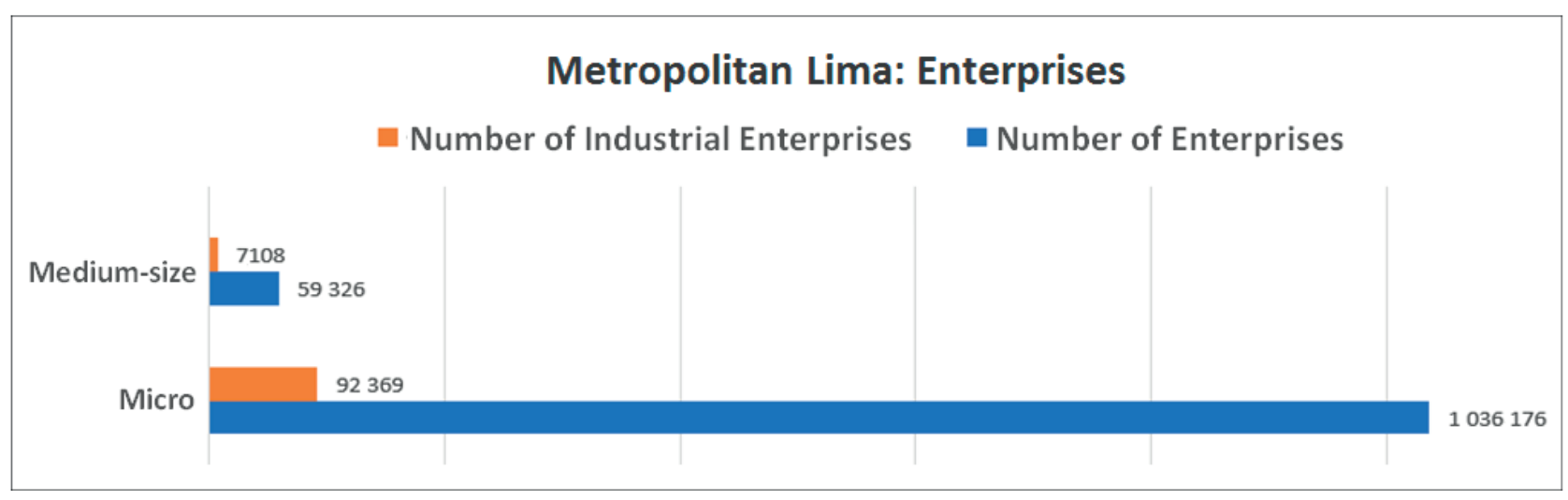

Figure 2. Study population.

Source: Prepared by the author. 
Table 3. Reliability of the Instrument.

\begin{tabular}{|c|c|c|c|c|c|}
\hline CSA & $\begin{array}{c}\text { Initial Cronbach's } \\
\text { alpha }\end{array}$ & $\begin{array}{c}\text { Final Cronbach's } \\
\text { Alpha }\end{array}$ & No. of initial items & $\begin{array}{l}\text { No. of deleted } \\
\text { items }\end{array}$ & No. of final items \\
\hline Vo & 0.726 & 0.914 & 8 & 2 & 6 \\
\hline V1 & 0.900 & 0.900 & 8 & & 8 \\
\hline V2 & 0.592 & 0.899 & 7 & 4 & 3 \\
\hline V3 & 0.906 & 0.906 & 3 & & 3 \\
\hline V4 & 0.716 & 0.756 & 4 & 1 & 3 \\
\hline V5 & 0.919 & 0.919 & 3 & & 3 \\
\hline V6 & 0.504 & 0.880 & 6 & 3 & 3 \\
\hline V7 & 0.796 & 0.796 & 3 & & 3 \\
\hline V8 & 0.824 & 0.824 & 3 & & 3 \\
\hline V9 & 0.660 & 0.660 & 3 & & 3 \\
\hline V10 & 0.681 & 0.737 & 5 & 2 & 3 \\
\hline V11 & 0.825 & 0.825 & 3 & & 3 \\
\hline V12 & 0.853 & 0.853 & 3 & & 3 \\
\hline$V 13$ & 0.889 & 0.889 & 4 & & 4 \\
\hline V14 & 0.751 & 0.801 & 4 & 1 & 3 \\
\hline TOTAL & & & 67 & 13 & 54 \\
\hline
\end{tabular}

Source: Prepared by the author.

\section{RESULTS}

\section{Final Survey}

The final survey included 62 items, of which 8 were control items and 54 research items, the latter divided into 14 critical attributes or constructs according to the model proposed for the research. A total of 190 valid surveys were obtained out of 210 completed. A link to the survey with a filling guide was sent to the responsible people within the company via digital communication (email, WhatsApp, other). Data was processed using the statistical software SPSS version 25.0 for Windows and Excel 2019 (Table 4).

As the questionnaire comprises 54 items and 14 constructs (critical attributes), it was necessary to subject each construct to an exploratory factor analysis to determine its appropriate configuration, as shown in Table 5.

As shown in Table 5, after carrying out the factor analysis of the constructs, it is determined that they are relevant. Furthermore, based on the original model, it is possible to create more homogeneous groups of constructs according to their correlation and interdependence.

The model of the new distribution of factors is shown in Figure 3.
On account of the type of model proposed, it is necessary to work on the basis of the 14 attributes. Table 4 shows the internal consistency of each attribute, all of which have a Cronbach's alpha coefficient greater than 0.81 , indicating that the internal consistencies are adequate.

Cronbach's alpha coefficient of the 54 items is 0.961 , which shows that they have a very good overall consistency (Table 6).

As shown in the table above, the general hypothesis is accepted, because the resulting significance level of the test is less than 0.05 . Also, the general hypothesis has a significant positive correlation at 0.770 .

\section{Final Model}

The final model is shown in Figure 4. It does not include the "environment" factor, since its critical attributes were rejected because they obtained significance levels greater than 0.05 in the result of the hypotheses.

\section{DISCUSSION}

The critical attribute management ICT experience (V14) is accepted because the significance level is less than 0.05 , with a weak negative correlation of 0.271 . 
Table 4. Reliability of the Final Instrument.

\begin{tabular}{llcc}
\hline CSA & Control Questions & Cronbach's Alpha & Items \\
\hline V0 & ICT adoption & 0.891 & 6 \\
V1 & Perceived benefits & 0.943 & 8 \\
V2 & Perceived complexity & 0.882 & 3 \\
V3 & Perceived security & 0.908 & 3 \\
V4 & Perceived cost & 0.914 & 3 \\
V5 & Technological readiness & 0.839 & 3 \\
V6 & Previous technological experience & 0.860 & 3 \\
V7 & Resistance to change & 0.995 & 3 \\
V8 & Organizational culture & 0.866 & 3 \\
V9 & Pressure from suppliers & 0.841 & 3 \\
V10 & Pressure from competitors & 0.814 & 3 \\
V11 & Government support & 0.857 & 3 \\
V12 & Senior management commitment & 0.988 & 3 \\
V13 & Managers' attitudes towards ICT adoption & 0.854 & 4 \\
V14 & Management ICT experience & 0.832 & 3 \\
V1-14 & & $\mathbf{0 . 9 6 1}$ & $\mathbf{5 4}$ \\
\hline
\end{tabular}

Source: Prepared by the author.

Table 5. Rotated Component Matrix.

\begin{tabular}{|c|c|c|c|c|c|c|}
\hline \multirow{2}{*}{ CSA } & \multirow{2}{*}{ Variable Description } & \multirow{2}{*}{ Items } & \multicolumn{4}{|c|}{ Item Correlation } \\
\hline & & & $1(\mathrm{~T})$ & 2(l) & $3(E)$ & $4(0)$ \\
\hline V9 & Pressure from suppliers & 3 & 0.973 & & & \\
\hline V8 & Organizational culture & 3 & 0.972 & & & \\
\hline V3 & Perceived safety & 3 & 0.967 & & & \\
\hline V4 & Perceived cost & 3 & 0.965 & & & \\
\hline V2 & Perceived complexity & 3 & 0.956 & & & \\
\hline $\mathrm{V} 1$ & Perceived benefits & 8 & 0.667 & & & \\
\hline V13 & Managers' attitudes towards ICT adoption & 4 & & 0.845 & & \\
\hline V12 & Top management commitment & 3 & & 0.752 & & \\
\hline V11 & Government support & 3 & & 0.694 & & \\
\hline V5 & Technological readiness of staff & 3 & & 0.587 & & \\
\hline V10 & Pressure from competitors & 3 & & & 0.860 & \\
\hline V6 & Previous technological experience & 3 & & & 0.798 & \\
\hline V7 & Resistance to change & 3 & & & & 0.853 \\
\hline V14 & Management ICT experience & 3 & & & & 0.740 \\
\hline
\end{tabular}

Extraction Method: Principal Component Analysis.

Rotation Method: Varimax with Kaizer Normalization.

Source: Prepared by the author.

For the critical attributes perceived benefits (V1), users' technological readiness (V5) and managers' attitudes towards ICT adoption (V13), a significant positive correlation is observed, as the resulting correlation ranges from 0.758 to 0.780 . Therefore, it can be said that there is a significant positive correlation between these attributes and the adoption of ICTs in industrial MSEs in Metropolitan Lima.
For the critical attributes perceived security (V3), perceived cost (V4), organizational culture (V8), pressure from suppliers (V9), government support (V11) and commitment of top management (V12), a medium positive correlation is observed, since the resulting correlation ranges between 0.553 and 0.611 . Thus, it can be said that there is a medium positive correlation between these hypotheses and 


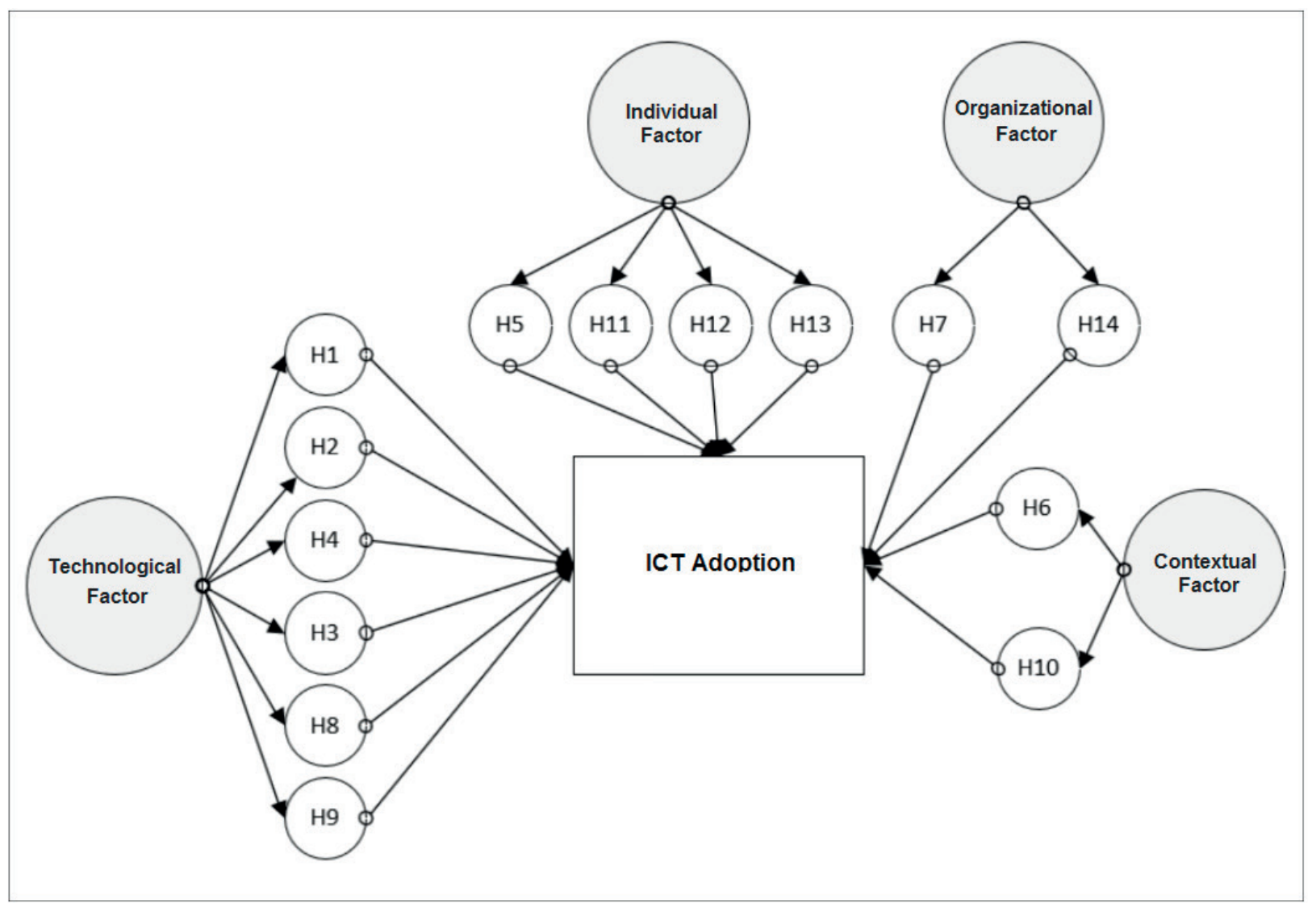

Figure 3. Model - Factor Analysis.

Source: Prepared by the author.

Table 6. Summary of Hypothesis Testing.

\begin{tabular}{|c|c|c|c|c|}
\hline & \multirow{2}{*}{$\begin{array}{l}\text { CSA } \\
\text { General hypothesis }\end{array}$} & \multirow{2}{*}{$\begin{array}{c}\text { Sig. } \\
0.000\end{array}$} & \multicolumn{2}{|r|}{ Correlation } \\
\hline & & & 0.770 & Significant positive correlation \\
\hline $\mathrm{V} 1$ & Perceived benefits & 0.000 & 0.780 & Significant positive correlation \\
\hline V2 & Perceived complexity & 0.000 & 0.670 & Medium positive correlation \\
\hline V3 & Perceived security & 0.000 & 0.611 & Medium positive correlation \\
\hline V4 & Perceived cost & 0.000 & 0.606 & Medium positive correlation \\
\hline V5 & Technological readiness of users & 0.000 & 0.758 & Significant positive correlation \\
\hline V6 & Previous technological experience & 0.622 & 0.036 & No correlation between variables \\
\hline V7 & Resistance to change & 0.544 & -0.044 & No correlation between variables \\
\hline V8 & Organizational culture & 0.000 & 0.586 & Medium positive correlation \\
\hline V9 & Pressure from suppliers & 0.000 & 0.597 & Medium positive correlation \\
\hline V10 & Pressure from competitors & 0.561 & 0.042 & No correlation between variables \\
\hline V11 & Government support & 0.000 & 0.564 & Medium positive correlation \\
\hline $\mathrm{V} 12$ & Senior management commitment & 0.000 & 0.553 & Medium positive correlation \\
\hline $\mathrm{V} 13$ & Managers' attitudes towards IT adoption & 0.000 & 0.772 & Significant positive correlation \\
\hline V14 & Management ICT experience & 0.000 & 0.271 & Medium negative correlation \\
\hline
\end{tabular}

Source: Prepared by the author. 


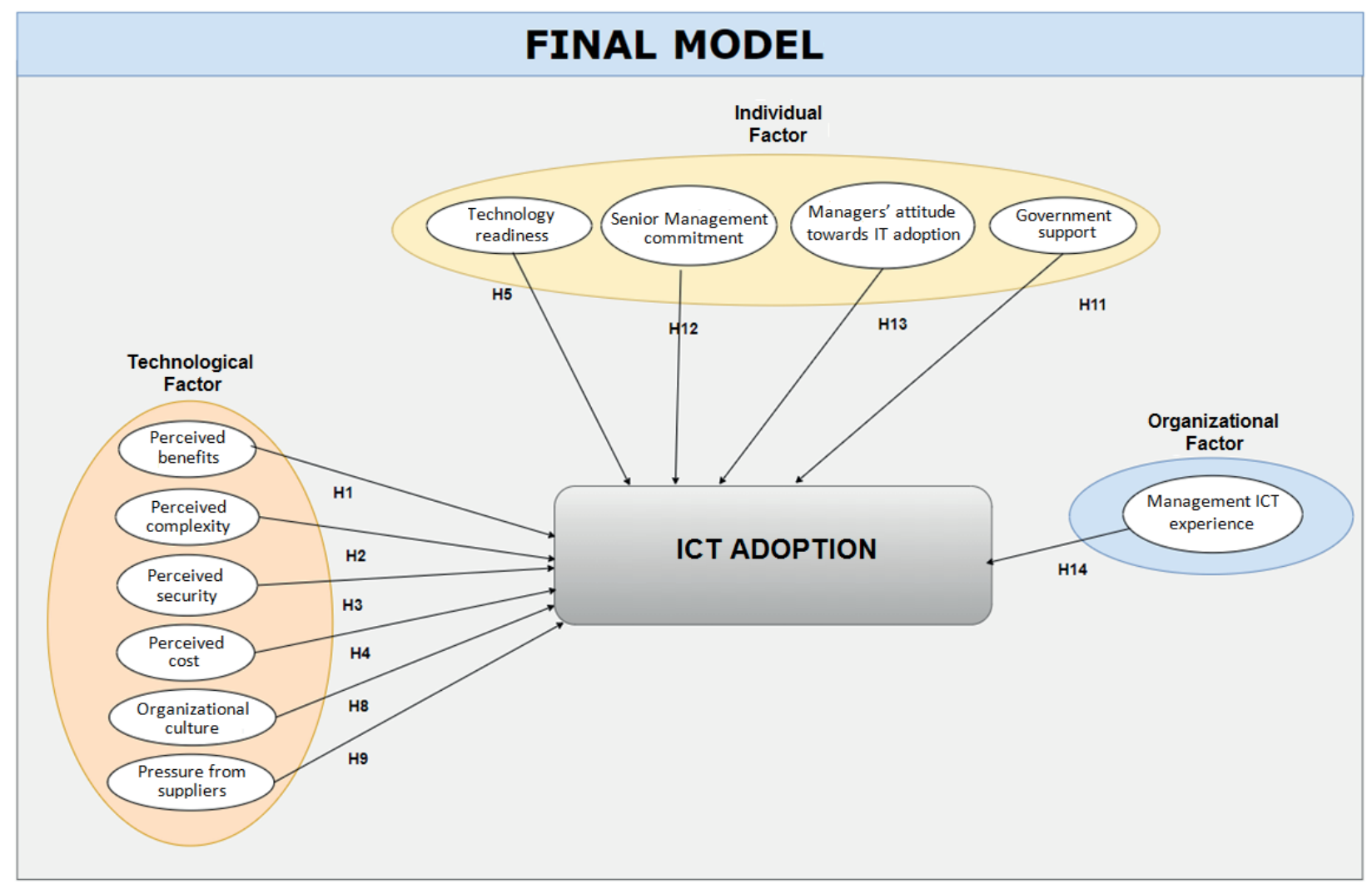

Figure 4. Model resulting from the accepted hypotheses.

Source: Prepared by the author.

the adoption of ICTs in the industrial MSEs in Metropolitan Lima.

On the other hand, critical attributes previous technological experience (V6), resistance to change (V7) and pressure from competitors (V10), are rejected because the significance level is greater than 0.05 .

The proposed conceptual framework allows researchers to empirically test and understand the critical factors for ICT adoption in MSEs in Metropolitan Lima. These factors are critical for the successful adoption of ICTs in MSEs.

Current ICT adoption patterns can be analyzed within this proposed framework and specific suggestions can be provided to improve the model.

This study also enhances the knowledge of the critical factors for ICT adoption in MSEs and helps managers to better assess the condition of the enterprise and the possible critical factors that would lead to the successful adoption of ICTs in their enterprises.
The findings of this research are also expected to contribute to the academic field, small and medium-sized enterprises, consulting firms and software providers, and government agencies.

\section{CONCLUSIONS}

Evidence in this research shows that there is a correlation between the factors proposed in the model and the adoption of ICTs in industrial MSEs in Metropolitan Lima, as shown in Table 6.

Critical factors "previous technological experience", "resistance to change" and "pressure from competitors" have no correlation with the adoption of ICTs in industrial companies in Metropolitan Lima.

Evidence from the research shows that the "environmental" factor disappears in the final model for the adoption of information and communication technologies in industrial companies in Metropolitan Lima, given that the proposed factors are not significant.

Fourteen critical factors were analyzed in this research; however, there may be others that are also 
important and were not considered for the development of this model.

\section{RECOMMENDATION}

The final model should be applied to the remaining Zones of APEIM 2020, by industry and nature of the products developed, in order to evaluate the model and analyze the results.

\section{REFERENCES}

[1] Alrawabdeh, W. (2014). Environmental Factors Affecting Mobile Commerce Adoption-An Exploratory Study on the Telecommunication Firms in Jordan. International Journal of Business and Social Science. 5 (8)., 151-164.

[2] Chang, S., Hung, S., Yen, D., \& Lee, P. (2010). Critical Factors of ERP Adoption for Small- and Medium- Sized Enterprises: An Empirical Study. Journal of Global Information Management (JGIM), 18(3), 82-106. Retrieved from http://doi.org/10.4018/jgim.2010070104

[3] Chau, N., \& Deng, H. (2018). Critical Determinants for Mobile Commerce Adoption in Vietnamese SMEs: A Conceptual Framework. Procedia Computer Science, 138, 433-440. Retrieved from https://doi.org/10.1016/j. procs.2018.10.061

[4] Chen, J., Windasari, N., \& Pai, R. (2013). Exploring E-Readiness on E-Commerce Adoption of SMEs: Case Study SouthEast Asia. IEEE International Conference on Industrial Engineering and Engineering Management. Retrieved from https://doi. org/10.1109/IEEM.2013.6962637

[5] Chiavenato, I. (2002). Introducción a la teoría general de la administración. 5. ${ }^{a}$ ed. . México D. F.: McGraw-Hill.

[6] Cuevas-Vargas, H., Estrada, S., \& LariosGómez, E. (2016). The effects of ICTs as innovation facilitators for a greater business performance. Evidence from Mexico. Procedia Computer Science, 91, 47-56. Retrieved from https://doi.org/10.1016/j.procs.2016.07.040

[7] Duan, X., Deng, H., \& Corbitt, B. (2012). What drives the adoption of electronic markets in Australian small-and-medium sized enterprises? - An empirical study. ACIS 2012 Proceedings. 20. Retrieved from https://aisel. aisnet.org/acis2012/20

[8] Gangwar, H., Date, H., \& Ramaswamy, R. (2015). Understanding determinants of cloud computing adoption using an integrated TAMTOE model. Journal of Enterprise Information Management, 28 (1), 107-130. Retrieved from https://doi.org/10.1108/JEIM-08-2013-0065

[9] Gibson, J., Ivancevich, J., Donelly, J., \& Konospake, R. (2006). Organizaciones: Comportamiento, Estructura y Procesos. 12. ed. México, D. F.: Mc-Graw Hill.

[10] Hassan, H. (2017). Organisational factors affecting cloud computing adoption in small and medium enterprises (SMEs) in service sector. Procedia Computer Science, 121, 976981. Retrieved from https://doi.org/10.1016/j. procs.2017.11.126

[11] Hernández, R., Fernández, C., \& Baptista, P. (2014). Metodología de la investigación (6a. ed.). México D.F.: McGraw-Hill.

[12] INEI (2020). PERÚ: Tecnologías de Información y Comunicación en las Empresas, 2017. Encuesta Económica Anual 2018. Retrieved from https://www.inei.gob.pe/media/ MenuRecursivo/publicaciones_digitales/Est/ Lib1719/libro.pdf

[13] Luiz, A., Costa, C., Dos Santos, J., \& Tsutsumi, M. (2020). Resistance to change as function of different physical-effort requirements in humans. Behavioural Processes, 176. Retrieved from https://doi.org/10.1016/j. beproc.2020.104123

[14] Morteza, G.,Arias-Aranda, D., \&Benitez-Amado, J. (2011). Adoption of e-commerce applications in SMEs. Industrial Management \& Data Systems, 111(8), 1238-1269. Retrieved from https://doi.org/10.1108/02635571111170785

[15] Oliveira, T., \& Martins, M. (2010). Understanding e-business adoption across industries in European countries. Industrial Management \& Data Systems, 110 (9), 1337-1354. Retrieved from https://doi. org/10.1108/02635571011087428

[16] Rahayu, R., \& Day, J. (2015). Determinant Factors of E-commerce Adoption by SMEs in Developing Country: Evidence from Indonesia. Procedia - Social and Behavioral Sciences, 195, 142 - 150. Retrieved from https://doi. org/10.1016/j.sbspro.2015.06.423

[17] Rauta, R., Gardas, B., Jha, M., \& Priyadarshinee, P. (2017). Examining the critical success factors of cloud computing adoption in the MSMEs by using ISM model. The Journal of High Technology Management Research. 28, 125- 
141. Retrieved from https://doi.org/10.1016/j. hitech.2017.10.004

[18] Rogers, E., \& Shoemaker, F. (1971). Communication of Innovations: A Cross-cultural Approach. Free Press, New York, NY.

[19] Singh, R., Luthra, S., Mangla, S., \& Uniyal, S. (2019). Applications of information and communication technology for sustainable growth of SMEs in India food industry. Resources, Conservation \& Recycling, 147, 10-18. Retrieved from https://doi.org/10.1016/j. resconrec.2019.04.014

[20] Sulaiman, A. (2000). The status of e-commerce applications in Malaysia. Information Technology for Development. 9, 153-161. Retrieved from https://doi.org/10.1080/026811 02.2000 .9525329

[21] Thong, J. (1999). An Integrated Model of Information Systems Adoption in Small Business. Journal of Management Information Systems, 15 (4), 187-214. Retrieved from https://doi.org/10.1080/07421222.1999.11518 227
[22] Tornatzky, L., \& Fleischer, M. (1990). The process of technology innovation. Lexington, MA: Lexington Books.

[23] Zhu, K., y Kenneth, K. (2005). Post-Adoption Variations in Usage and Value of E-Business by Organizations. Information Systems Research. 16 (1), 61-84. Retrieved from http://dx.doi. org/10.1287/isre.1050.0045

[24] Zhu, K., Kraemer, K., \& Xu, S. (2006). The Process of Innovation Assimilation by Firms in Different Countries: A Technology Diffusion Perspective on E-Business. Management Science, 52(10), 1557-1576. Retrieved from https://doi.org/10.1287/mnsc.1050.0487 